\title{
Sum Transmission Rate Maximization Based Cooperative Spectrum Sharing with Both Primary and Secondary QoS-Guarantee
}

\author{
Weidang Lu ${ }^{1,2}$, Yufei Zhu ${ }^{1,2}$, Mengyun Wang ${ }^{1,2}$, Hong Peng ${ }^{1,2}$, Xin Liu ${ }^{3}$ \\ ${ }^{1}$ College of Information Engineering, Zhejiang University of Technology \\ Hangzhou, Zhejiang 310023 - China \\ [e-mail: \{luweid, ph\}@zjut.edu.cn] \\ ${ }^{2}$ Zhejiang Provincial Key Laboratory of Communication Networks and Applications, Zhejiang University of \\ Technology, Hangzhou, Zhejiang 310023 - China \\ ${ }^{3}$ College of Astronautics, Nanjing University of Aeronautics and Astronautics \\ Nanjing, Jiangsu 211106 - China \\ [e-mail: liuxinstar1984@auaa.edu.cn] \\ *Corresponding author: Weidang Lu
}

Received October 14, 2015; revised January 3, 2016; accepted March 30, 2016;

published May 31, 2016

\begin{abstract}
In this paper, we propose a sum transmission rate maximization based cooperative spectrum sharing protocol with quality-of-service (QoS) support for both of the primary and secondary systems, which exploits the situation when the primary system experiences a weak channel. The secondary transmitter $S T_{b}$ which provides the best performance for the primary and secondary systems is selected to forward the primary signal. Specifically, $S T_{b}$ helps the primary system achieve the target rate by using a fraction of its power to forward the primary signal. As a reward, it can gain spectrum access by using the remaining power to transmit its own signal. We study the secondary user selection and optimal power allocation such that the sum transmission rate of primary and secondary systems is maximized, while the QoS of both primary and secondary systems can be guaranteed. Simulation results demonstrate the efficiency of the proposed spectrum sharing protocol and its benefit to both primary and secondary systems.
\end{abstract}

Keywords: spectrum sharing; cooperative transmission; decode-and-forward relaying; power allocation; QoS

This work was supported by China National Science Foundation under Grand No. 61402416 and 61303235, Natural Science Foundation of Zhejiang Province under Grant No. LQ14F010003, LQ14H180001 and LQ14F020005, NSFC-Zhejiang Joint Fund for the Integration of Industrialization and Informatization under grant No. U1509219, Natural Science Foundation of Jiangsu Province under Grant No. BK20140828 and the Scientific Foundation for the Returned Overseas Chinese Scholars of State Education Ministry. 


\section{Introduction}

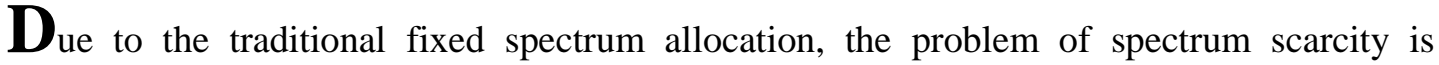
becoming sharply prominent with the increasing demand of wireless users and applications. Spectrum measurements indicate that the licensed spectrum is largely under-utilized ranging from as low as $15 \%$ to $85 \%$, which varies significantly over time and geography $[1,2]$. Cognitive radio (CR) has been proposed as an effective way to enable an efficient utilization of the licensed spectrum by allowing the unlicensed (secondary) systems to operate in licensed spectrum bands of the licensed (primary) systems while the interference caused by the secondary systems below the tolerable levels [3-6].

Most spectrum sharing protocols are under the condition that the primary direct transmission is good enough, such that the primary system can tolerate additional interference caused by the secondary system [7-12]. In this way, the secondary system will be permitted to access the primary spectrum and works simultaneously with the primary system if it does not affect the primary QoS. [7] and [8] studied the optimal power allocation which maximizes the secondary achievable rate subject to the interference power constraint at the primary receiver, in order to protect primary transmission. In [9], a robust power control scheme is proposed to guarantee the interference temperature of the PUs through operating in the network-centric manner, and keeps the fairness between the SUs through link gain pricing. [10] studied optimization algorithms for decision making to optimize radio resource usage in centralized and decentralized cognitive wireless networks. [11] studied the weighted sum rate maximization problem which maximizes the total throughput also known as the sum rates of all the users while avoiding the interference of unlicensed band secondary users from overwhelming the licensed band primary users. However, the above spectrum sharing strategies cannot work when the direct transmission link of primary system is not good enough to guarantee its reliable transmission. In this case, it is effective to apply cooperative diversity in cognitive systems [13].

Cooperative relaying predominates in spectrum sharing field because of its ability to take advantage of user diversity and improve capacity and reliability in wireless network [14-16], especially when the primary system cannot achieve its QoS, i.e., target rate, through the direct transmission link. [17-21] studied different cooperation forms in cognitive radios. The use of cooperative relaying for the secondary transmissions with a primary user's QoS constraint is discussed in [18], for which a diversity-multiplexing trade-off is developed. [19] proposed a spectrum sharing protocol based on cooperative decode-and-forward relaying where the outage probability of primary system will not be larger. [20] proposed new robust CR cooperative relay beamformers where either the total relay transmit power or the cognitive destination signal-to-interference-and-noise ratio is optimized subject to a constraint on the primary receiver outage probability. [21] proposed jointly optimizing the beamforming vector and power allocation for the secondary transmitter in order to maximize the rate for the secondary system while meeting the rate requirement for the primary system.

Most of existing works concentrate on secondary transmission rate maximization with only considering the primary system achieves its QoS. However, some service of secondary system, e.g., multimedia applications, may also need QoS guarantee. In this paper, we propose a sum transmission rate maximization based cooperative spectrum sharing protocol, in which both the QoS of primary and secondary systems can be guaranteed. Specifically, the primary signal 
is forwarded by the selected secondary transmitter with a fraction of its power to achieve the target rate. As a reward, it can achieve its own target rate by using the remaining power to transmit its own signal. We study the secondary user selection and optimal power allocation such that the sum transmission rate of primary and secondary systems is maximized, while the QoS of both primary and secondary system can be guaranteed.

The main contributions of this paper are summarized as follows. First, a sum transmission rate maximization based cooperative spectrum sharing protocol is proposed, where both the primary and secondary systems can achieve their target rate. Second, secondary user selection and optimal power allocation is derived in the proposed spectrum sharing protocol, such that the sum transmission rate of primary and secondary systems is maximized, while the QoS of both primary and secondary system can be guaranteed. Finally, Simulation results are shown to demonstrate the efficiency of the proposed spectrum sharing protocol.

The left of this paper is organized as follows. The system model and the proposed spectrum sharing protocol are presented in Section 2. And in Section 3, we analyze the optimal power allocation. Computer simulation results are demonstrated in Section 4 to illustrate the performance of the proposed spectrum sharing strategy. Finally we make a conclusion in Section V.

\section{System Model}

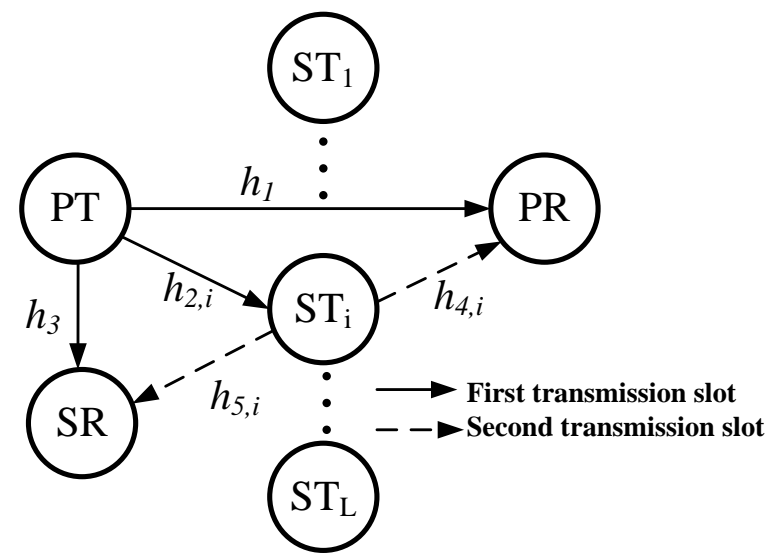

Fig. 1. System model

We consider an underlay cognitive radio wireless network and the system configuration is shown in Fig. 1. The primary system consists of a primary transmitter (PT) and a primary receiver (PR), which supports relaying function [17-19]. The secondary system comprises of $L$ secondary transmitters $\left(S T_{i}\right), i \in\{1,2,3, \ldots, L\}$ and a common secondary receiver (SR), which is seeking to exploit possible transmission opportunities. We assume that the secondary system can emulate the radio protocols and system parameters of the primary system.

The channels over links $P T \rightarrow P R, P T \rightarrow S T_{i}, P T \rightarrow S R, S T_{i} \rightarrow P R$, and $S T_{i} \rightarrow S R$ are modeled on Rayleigh flat fading with channel coefficients denoted by $h_{1}, h_{2, i}, h_{3}, h_{4, i}$ and $h_{5, i}$, respectively. We assume all channels obey complex Gaussian distribution, i.e., $h_{j} \sim C N\left(0, d_{j}^{-v}\right), j=1,3$ and $h_{n, i} \sim C N\left(0, d_{n, i}^{-v}\right), n=2,4,5$, where $v$ is the path loss exponent and $d_{j}, d_{n, i}$ are the normalized distance between the respective transmitters and receivers. This 
normalization is done with respect to the distance between PT and PR, i.e., $d_{1}=1$. The instantaneous channel gain is denoted as $r_{j}=\left|h_{j}\right|^{2}$ or $r_{n, i}=\left|h_{n, i}\right|^{2}$. The variances of the additive white Gaussian noise (AWGN) at all receivers are assumed to be identical and denoted as $\sigma^{2}$. The primary and secondary signals are denoted as $x_{p}$ and $x_{s}$ with zero mean and unit variance, i.e., $E\left\{x_{p}^{*} x_{p}\right\}=E\left\{x_{s}^{*} x_{s}\right\}=1$. The transmit power at PT and ST is denoted as $P_{p}$ and $P_{s}$, respectively.

\section{Secondary User Selection and Performance Analysis}

\subsection{Secondary user selection}

When $P T \rightarrow P R$ link experiences a poor link quality due to path loss, shadowing, or interference (e.g., when the primary user moves to the cell edge in a primary cellular network), causing the achievable primary rate to fall below a target rate. PT will seek cooperation from neighboring secondary transmitter to enhance its transmission performance by sending out a request-to-cooperate (RTC) signal, in which the primary target rate is embedded. This RTC will be responded by PR with an acknowledge-to-cooperation (ATC) signal.

Upon receiving RTC and ATC signals, $S T_{i}$ is able to estimate channel gains $r_{2, i}$ and $r_{4, i}$. Accordingly, $S T_{i}$ decides whether the primary and secondary systems can achieve their target rates when $S T_{i}$ accesses to the primary spectrum by calculating the maximum instantaneous rate, $R_{p, i}^{\max }$ and $R_{s, i}^{\max }$, when $S T_{i}$ uses all of its power to relay primary and secondary signals, respectively.

$$
\begin{aligned}
R_{p, i}^{\max } & =\min \left(R_{1, i}^{\max }, R_{2, i}^{\max }\right) \\
R_{s, i}^{\max } & =\frac{1}{2} \log _{2}\left(1+\frac{P_{s} r_{5, i}}{\sigma^{2}}\right)
\end{aligned}
$$

where $R_{1, i}^{\max }=\frac{1}{2} \log _{2}\left(1+\frac{P_{p} r_{2, i}}{\sigma^{2}}\right)$ and $R_{2, i}^{\max }=\frac{1}{2} \log _{2}\left(1+\frac{P_{s} r_{4, i}}{\sigma^{2}}+\frac{P_{p} r_{1}}{\sigma^{2}}\right)$ are the maximum achievable rates of $P T \rightarrow S T_{i}$ and $S T_{i} \rightarrow P R$ links, respectively.

We denote $D=\left\{i \in L, R_{p, i}^{\max } \geq R_{P T} \cap R_{s, i}^{\max } \geq R_{S T}\right\}$. It is clear that when $S T_{i}, \forall i \in D$, accesses to the primary spectrum, both primary and secondary system can achieve their target rates.

Each secondary transmitter $S T_{i}, \forall i \in D$, now starts a countdown with initial value

$$
T_{i}=\frac{C}{R_{p, i}^{\max }+R_{s, i}^{\max }}
$$

where $\mathrm{C}$ is a normalization constant. It is obviouse from (1), (2) and (3) that $S T_{b}$, where $b=\arg \min T_{i}$, will provide the maximum sum transmission rate for the primary and secondary systems.

Therefore the best secondary transmitter $S T_{b}$ has its timer reduced to zero first, and it broadcasts a confirm-to-cooperate (CTC) signal to identify its presence. All other $S T_{i}$, $i \in D \backslash\{b\}$ will back off after hearing CTC. Upon receiving CTC, the primary system is aware that its target rate can be achieved through cooperation and it switches into a two-phase DF relaying mode with $S T_{b}$ being the relay node. Otherwise, the primary system do not receive 
CTC, i.e., $|D|=0$, the primary system will work with direct transmission and the secondary system will remain silent.

\subsection{Performance analysis}

In this section, we will derive the achievable rate of primary and secondary systems with $S T_{b}$ accessing to the primary spectrum by the following two-slot cooperative transmission.

In the first transmission slot, PT broadcasts its signal. The achievable rates of $P T \rightarrow P R$, $P T \rightarrow S T_{b}, P T \rightarrow S R$ links can be given as

$$
\begin{aligned}
& R_{11}=\frac{1}{2} \log _{2}\left(1+\frac{P_{p} r_{1}}{\sigma^{2}}\right) \\
& R_{12}=\frac{1}{2} \log _{2}\left(1+\frac{P_{p} r_{2, b}}{\sigma^{2}}\right) \\
& R_{13}=\frac{1}{2} \log _{2}\left(1+\frac{P_{p} r_{3}}{\sigma^{2}}\right)
\end{aligned}
$$

Then $S T_{b}$ generates a composite signal $z_{s}$ by linearly combining the regenerated $x_{p}$ with power $\alpha P_{s}(0<\alpha<1)$ and the secondary signal $x_{s}$ with power $(1-\alpha) P_{s}$. Thus $z_{s}=\sqrt{\alpha P_{s}} x_{p}+\sqrt{(1-\alpha) P_{s}} x_{s}$.

In the second transmission slot, $S T_{b}$ broadcasts $z_{s}$. Thus, the achievable rate at PR can be given as

$$
R_{21}=\frac{1}{2} \log _{2}\left(1+\frac{\alpha P_{s} r_{4, b}}{(1-\alpha) P_{s} r_{4, b}+\sigma^{2}}+\frac{P_{s} r_{1}}{\sigma^{2}}\right)
$$

The achievable rate of $P T \rightarrow S R$ link is obtained depending on whether SR can successfully decode the primary signal in the first transmission slot

where $R_{P T}$ is primary target rate.

$$
R_{22}= \begin{cases}\frac{1}{2} \log _{2}\left(1+\frac{(1-\alpha) P_{s} r_{5, b}}{\alpha P_{s} r_{5}+\sigma^{2}}\right) & R_{13}<R_{P T} \\ \frac{1}{2} \log _{2}\left(1+\frac{(1-\alpha) P_{s} r_{5, b}}{\sigma^{2}}\right) & R_{13} \geq R_{P T}\end{cases}
$$

Thus, the primary and secondary achievable rate over two transmission slots can be given as

$$
\begin{gathered}
R_{p}=\min \left\{R_{12}, R_{21}\right\} \\
R_{s}=R_{22}
\end{gathered}
$$

\section{Optimal Power Allocation}

In this section, we seek optimal power allocation $\alpha$ to maximize the sum transmission rate of primary and secondary systems under the condition that both of the primary and secondary systems achieve their target rates. This optimization problem can be formulated as

$$
\max _{\alpha}\left(R_{p}+R_{s}\right)
$$

subject to 


$$
\left\{\begin{array}{l}
R_{p} \geq R_{P T} \\
R_{s} \geq R_{S T} \\
0<\alpha<1
\end{array}\right.
$$

where $R_{S T}$ is the target rate of secondary system, which can be chosen depending on the service of the secondary system. The different importance data rate for PR and SR can be reflected by choosing different target rate $R_{P T}$ and $R_{S T}$.

From (9), we can find that $R_{p}$ may have two different values

$$
R_{p}= \begin{cases}R_{12} & \alpha>\alpha_{0} \\ R_{21} & \alpha \leq \alpha_{0}\end{cases}
$$

where $\alpha_{0}=\frac{P_{p}\left(r_{2, b}-r_{1}\right)\left(P_{s} r_{4, b}+\sigma^{2}\right)}{P_{s} r_{4, b}\left[P_{p}\left(r_{2, b}-r_{1}\right)+\sigma^{2}\right]}$.

Thus, to satisfy the first condition of (12), we can obtain

$$
\begin{cases}\frac{1}{2} \log _{2}\left(1+\frac{P_{p} r_{2, b}}{\sigma^{2}}\right) \geq R_{P T} & \alpha>\alpha_{0} \\ \alpha \geq \frac{R_{10}\left(P_{s} r_{4, b}+\sigma^{2}\right)}{P_{s} r_{4, b}\left(1+R_{10}\right)} \triangleq \alpha_{\min } & \alpha \leq \alpha_{0}\end{cases}
$$

where $R_{10}=2^{2 R_{P T}}-1-\frac{P_{p} r_{1}}{\sigma^{2}}$. It is easy to find that $\alpha_{\text {min }}$ is larger than 0 . We can also find that the primary achievable rate should be larger than its target rate, i.e., $\frac{1}{2} \log _{2}\left(1+\frac{P_{s} r_{4, b}}{\sigma^{2}}+\frac{P_{p} r_{1}}{\sigma^{2}}\right)>R_{P T}$, when the secondary system uses all of its power to forward the primary signal. Otherwise, the secondary system cannot help the primary system achieve the target rate and it will not have chance to be allowed to access the primary spectrum. Thus, we can obtain $\frac{P_{s} r_{4, b}}{\sigma^{2}}>R_{10}$, then it is easy to find $\alpha_{\min }<1$.

From (8), we can observe that $R_{s}$ has two different values. Thus, we will obtain the optimal power allocation in the following two cases with different values of $R_{s}$.

Case A: when $R_{13} \geq R_{P T}, R_{s}=\frac{1}{2} \log _{2}\left(1+\frac{(1-\alpha) P_{s} r_{5, b}}{\sigma^{2}}\right)$.

To satisfy the second condition of (12), we can obtain

$$
\alpha \leq 1-\frac{R_{20} \sigma^{2}}{P_{s} r_{5, b}} \triangleq \alpha_{\max }
$$

where $R_{20}=2^{2 R_{S T}}-1$. It is easy to find that $\alpha_{\max }$ is smaller than 1 . We can also find that the secondary achievable rate should be larger than its target rate, i.e., $\frac{1}{2} \log _{2}\left(1+\frac{P_{s} r_{5, b}}{\sigma^{2}}\right)>R_{S T}$, when the secondary system uses all of its power to transmit its own signal. Otherwise, the secondary system will not be willing to help forward the primary signal. Thus, we can obtain $\frac{P_{s} r_{5, b}}{\sigma^{2}}>R_{20}$, then it is easy to find $0<\alpha_{\max }<1$. 
Assume $f(\alpha)=\frac{1}{2} \log _{2}\left(1+\frac{\alpha P_{s} r_{4, b}}{(1-\alpha) P_{s} r_{4, b}+\sigma^{2}}+\frac{P_{p} r_{1}}{\sigma^{2}}\right)$, we can find that $f(\alpha)$ monotonically increases with $\alpha$. From the above analysis, as we know $f\left(\alpha_{0}\right)=R_{12}, f\left(\alpha_{\min }\right)=R_{P T}$, and $R_{12}$ should be larger than $R_{P T}$, then we can obtain $\alpha_{0} \geq \alpha_{\min }$.

Thus, the optimal $\alpha$ can be obtained by analyzing the relative values of $\alpha_{0}$ and $\alpha_{\max }$ as follows.

a) Under the condition of $\alpha_{0} \geq \alpha_{\max }$

We can obtain $\alpha \leq \alpha_{0}$, thus $R_{p} \leq R_{21}$. Assume $L(\alpha)=R_{p}+R_{s}$, then

$$
L(\alpha)=R_{21}+R_{22}=\frac{1}{2} \log _{2}(Y(\alpha))
$$

where

$$
Y(\alpha)=\left(\frac{P_{s} r_{4, b}+\sigma^{2}}{(1-\alpha) P_{s} r_{4, b}+\sigma^{2}}\right)\left(1+\frac{(1-\alpha) P_{s} r_{5, b}}{\sigma^{2}}\right)
$$

Obviously, $L(\alpha)$ and $Y(\alpha)$ have the same monotonicity. Take the first derivation of $Y(\alpha)$ with $\alpha$, we can obtain

$$
Y^{\prime}(\alpha)=\frac{A(\alpha)}{\sigma^{4}\left[(1-\alpha) P_{s} r_{4, b}+\sigma^{2}\right]^{2}}
$$

where

$$
A(\alpha)=P_{s}\left(P_{s} r_{4, b}+\sigma^{2}\right)\left(r_{4, b}-r_{5, b}\right)-P_{p} P_{s} r_{1} r_{5, b}\left[(1-\alpha) P_{s} r_{4, b}+\sigma^{2}\right]^{2}
$$

From (18), we can find that the monotonicity of $Y(\alpha)$ depends on $A(\alpha)$ as the denominator of $Y^{\prime}(\alpha)$ is always positive. We can find that $A(\alpha)$ is a quadratic convex function of $\alpha$ and its symmetry axis equals to $\frac{P_{s} r_{4, b}+\sigma^{2}}{P_{s} r_{4, b}}$, which is larger than 1 . Thus, we can obtain that $A(\alpha)$ monotonically increase with $\alpha$ when $\alpha \in[0,1]$. Then, the optimal $\alpha$ can be obtained by analyzing the relative value of $A\left(\alpha_{\max }\right)$ and 0 .

1) when $A\left(\alpha_{\max }\right) \leq 0$

We can obtain $Y^{\prime}(\alpha) \leq 0$, when $\alpha \in\left[\alpha_{\min }, \alpha_{\max }\right]$, which indicates that $L(\alpha)$ monotonically decreases with $\alpha$ in this region. Thus, the optimal value of $\alpha$ can be obtained as

$$
\alpha^{*}=\alpha_{\min }=\frac{R_{10}\left(P_{s} r_{4, b}+\sigma^{2}\right)}{P_{s} r_{4, b}\left(1+R_{10}\right)}
$$

2) when $A\left(\alpha_{\max }\right)>0$

We can obtain that there must exist one and only one station point when $\alpha \in\left[-\infty, \alpha_{\max }\right]$, as $A(\alpha)$ is a quadratic convex function. No matter the station point is in $\left[\alpha_{\min }, \alpha_{\max }\right]$ or not, the optimal value of $\alpha$ will be obtained at $\alpha_{\min }$ or $\alpha_{\max }$. Specifically, the optimal value of $\alpha$ can be obtained as 


$$
\alpha^{*}= \begin{cases}\alpha_{\min }=\frac{R_{10}\left(P_{s} r_{4, b}+\sigma^{2}\right)}{P_{s} r_{4, b}\left(1+R_{10}\right)} & L\left(\alpha_{\min }\right) \geq L\left(\alpha_{\max }\right) \\ \alpha_{\text {max }}=1-\frac{R_{20} \sigma^{2}}{P_{s} r_{5, b}} & L\left(\alpha_{\text {min }}\right)<L\left(\alpha_{\text {max }}\right)\end{cases}
$$

\section{b) Under the condition of $\alpha_{0}<\alpha_{\max }$}

When $\alpha \in\left[\alpha_{\min }, \alpha_{0}\right]$, we can obtain $\alpha \leq \alpha_{0}$, thus $R_{p}=R_{21}$. The optimal value of $\alpha$ can be obtained with the same analysis as in a).

When $\alpha \in\left[\alpha_{0}, \alpha_{\max }\right]$, we can obtain $\alpha>\alpha_{0}$, thus $R_{p}=R_{12}$, then

$$
L(\alpha)=R_{12}+R_{22}=\frac{1}{2} \log _{2}\left(1+\frac{P_{p} r_{2, b}}{\sigma^{2}}\right)\left(1+\frac{(1-\alpha) P_{s} r_{5, b}}{\sigma^{2}}\right)
$$

It is easy to find that $L(\alpha)$ is a monotonically decreasing function of $\alpha$. Thus, the optimal value of $\alpha$ equals to $\alpha_{0}$.

Thus, the optimal $\alpha^{*}$ in Case A is summarized in Table 1.

\begin{tabular}{|c|c|c|c|c|}
\hline \multicolumn{4}{|c|}{ Condition } & $\alpha^{*}$ \\
\hline \multicolumn{5}{|l|}{ a) $\alpha_{0} \geq \alpha_{\max }$} \\
\hline \multirow{3}{*}{$\alpha \in\left[\alpha_{\min }, \alpha_{\max }\right]$} & 1) & \multicolumn{2}{|l|}{$A\left(\alpha_{\max }\right) \leq 0$} & $\alpha_{\min }$ \\
\hline & \multirow{2}{*}{ 2) } & \multirow[t]{2}{*}{$A\left(\alpha_{\max }\right)>0$} & $L\left(\alpha_{\min }\right) \geq L\left(\alpha_{\max }\right)$ & $\alpha_{\min }$ \\
\hline & & & $L\left(\alpha_{\min }\right)<L\left(\alpha_{\max }\right)$ & $\alpha_{\max }$ \\
\hline \multicolumn{5}{|l|}{ b) $\alpha_{0}<\alpha_{\max }$} \\
\hline \multirow{3}{*}{$\alpha \in\left[\alpha_{\min }, \alpha_{0}\right]$} & 1) & \multicolumn{2}{|l|}{$A\left(\alpha_{0}\right) \leq 0$} & $\alpha_{\min }$ \\
\hline & \multirow{2}{*}{\multicolumn{2}{|c|}{ 2) $\quad A\left(\alpha_{0}\right)>0$}} & $L\left(\alpha_{\min }\right) \geq L\left(\alpha_{0}\right)$ & $\alpha_{\min }$ \\
\hline & & & $L\left(\alpha_{\min }\right)<L\left(\alpha_{0}\right)$ & $\alpha_{0}$ \\
\hline \multicolumn{4}{|l|}{$\alpha \in\left[\alpha_{0}, \alpha_{\max }\right]$} & $\alpha_{0}$ \\
\hline
\end{tabular}

Table 1. Optimal $\alpha^{*}$ in Case A

Case B: when $R_{13}<R_{P T}, R_{s}=\frac{1}{2} \log _{2}\left(1+\frac{(1-\alpha) P_{s} r_{5, b}}{\alpha P_{s} r_{5, b}+\sigma^{2}}\right)$.

To satisfy the second condition of (12), we can obtain

$$
\alpha \leq \frac{P_{s} r_{5, b}-R_{20} \sigma^{2}}{P_{s} r_{5, b}\left(1+R_{20}\right)} \triangleq \alpha_{\max }
$$

It is easy to find that $\alpha_{\max }$ is smaller than 1 . In the above we have already known that $\frac{1}{2} \log _{2}\left(1+\frac{P_{s} r_{5, b}}{\sigma^{2}}\right)>R_{S T}$, then we can obtain $0<\alpha_{\max }<1$.

We have already known that $\alpha_{0} \geq \alpha_{\min }$. Thus the optimal $\alpha$ can be obtained by analyzing the relative values of $\alpha_{0}$ and $\alpha_{\max }$ as follows.

a) Under the condition of $\alpha_{0} \geq \alpha_{\max }$.

We can obtain $\alpha \leq \alpha_{0}$, thus $R_{p} \leq R_{21}$. Then 
where

$$
L(\alpha)=\frac{1}{2} \log _{2}(G(\alpha))
$$

$$
G(\alpha)=\frac{1}{2} \log _{2}\left(\frac{P_{s} r_{5, b}+\sigma^{2}}{\alpha P_{s} r_{5, b}+\sigma^{2}}\left(\frac{P_{s} r_{4, b}+\sigma^{2}}{(1-\alpha) P_{s} r_{4, b}+\sigma^{2}}+\frac{P_{p} r_{5, b}}{\sigma^{2}}\right)\right)
$$

Take the first derivation of $G(\alpha)$ with $\alpha$, we can obtain

$$
G^{\prime}(\alpha)=\frac{B(\alpha)}{C(\alpha)}
$$

where

$$
\begin{gathered}
B(\alpha)=-P_{s}^{2} r_{4, b} r_{5, b}\left(\frac{\alpha^{2} P_{s} r_{4, b} P_{p} r_{1}}{\sigma^{2}}-2 \alpha\left(1+\frac{P_{p} r_{1}}{\sigma^{2}}\right)\left(P_{s} r_{4, b}+\sigma^{2}\right)\right) \\
+P_{s} r_{4, b}\left(P_{s} r_{4, b}+\sigma^{2}\right) \sigma^{2}-P_{s} r_{5, b}\left(1+\frac{P_{p} r_{1}}{\sigma^{2}}\right)\left(P_{s} r_{4, b}+\sigma^{2}\right)^{2} \\
C(\alpha)=\left(\left(1+\frac{P_{p} r_{1}}{\sigma^{2}}\right)(1-\alpha) P_{s} r_{4, b}+\sigma^{2}\right)^{2}+\alpha P_{s} r_{4, b}\left((1-\alpha) P_{s} r_{4, b}+\sigma^{2}\right)\left(\alpha P_{s} r_{5, b}+\sigma^{2}\right)
\end{gathered}
$$

From (26), we can find that the monotonicity of $G(\alpha)$ depends on $B(\alpha)$ as $C(\alpha)$ is always positive. We can find that $B(\alpha)$ is a quadratic convex function $\alpha$, and the symmetry axis equals to $\frac{\left(P_{s} r_{4, b}+\sigma^{2}\right)\left(P_{p} r_{1}+\sigma^{2}\right)}{P_{s} r_{4, b} P_{p} r_{1}}$, which is larger than 1 . Thus, we can obtain that $B(\alpha)$ monotonically increases with $\alpha$ when $\alpha \in[0,1]$. The optimal value of $\alpha$ can be obtained with the similar method in case A.a.

\section{b) Under the condition of $\alpha_{0}<\alpha_{\max }$.}

When $\alpha \in\left[\alpha_{\min }, \alpha_{0}\right]$, we can obtain $\alpha \leq \alpha_{0}$, thus $R_{p}=R_{21}$. The optimal value of $\alpha$ can be obtained with the same analysis as in a).

When $\alpha \in\left[\alpha_{0}, \alpha_{\max }\right]$, we can obtain $\alpha>\alpha_{0}$, thus $R_{p}=R_{12}$, then

$$
L(\alpha)=\frac{1}{2} \log _{2}\left(1+\frac{P_{p} r_{2, b}}{\sigma^{2}}\right)\left(1+\frac{(1-\alpha) P_{s} r_{5, b}}{\alpha P_{s} r_{5, b}+\sigma^{2}}\right)
$$

\begin{tabular}{|c|c|c|c|c|}
\hline \multicolumn{4}{|c|}{ Condition } & $\alpha^{*}$ \\
\hline \multicolumn{5}{|l|}{ a) $\alpha_{0} \geq \alpha_{\text {max }}$} \\
\hline \multirow{3}{*}{$\alpha \in\left[\alpha_{\min }, \alpha_{\max }\right]$} & 1) & $B\left(\alpha_{\max }\right) \leq 0$ & & $\alpha_{\min }$ \\
\hline & & \multirow[t]{2}{*}{$B\left(\alpha_{\max }\right)>0$} & $L\left(\alpha_{\min }\right) \geq L\left(\alpha_{\max }\right)$ & $\alpha_{\min }$ \\
\hline & & & $L\left(\alpha_{\min }\right)<L\left(\alpha_{\max }\right)$ & $\alpha_{\max }$ \\
\hline
\end{tabular}

It is easy to find that $L(\alpha)$ is a monotonically decreasing function of $\alpha$. Thus, the optimal value of $\alpha$ equals to $\alpha_{0}$.

Thus, the optimal $\alpha^{*}$ in Case B is summarized in Table 2.

Table 2. Optimal $\alpha^{*}$ in Case B 


\begin{tabular}{|c|ll|c|c|}
\hline \multirow{3}{*}{$\alpha \in\left[\alpha_{\min }, \alpha_{0}\right]$} & $1) \quad B\left(\alpha_{0}\right) \leq 0$ & $\alpha_{\min }$ \\
\cline { 2 - 4 } & $2)$ & $B\left(\alpha_{0}\right)>0$ & $L\left(\alpha_{\min }\right) \geq L\left(\alpha_{0}\right)$ & $\alpha_{\min }$ \\
\cline { 2 - 4 } & & $L\left(\alpha_{\min }\right)<L\left(\alpha_{0}\right)$ & $\alpha_{0}$ \\
\hline$\alpha \in\left[\alpha_{0}, \alpha_{\max }\right]$ & & $\alpha_{0}$ \\
\hline
\end{tabular}

\section{Simulation Results and Discussions}

In this section, simulation results are presented to demonstrate the performance of the proposed spectrum sharing protocol. We consider $\mathrm{PT}, \mathrm{PR}, S T_{b}$ and $\mathrm{SR}$ are in a two-dimensional X-Y plane, where PT and PR are located at points $(0,0)$ and $(1,0)$, respectively, thus $d_{1}=1$. The channel over $P T \rightarrow P R$ link experiences $20 \mathrm{~dB}$ fading. $S T_{b}$ moves on the positive $\mathrm{X}$ axis, its coordinates are $\left(d_{2}, 0\right)$. SR is located at $\left(d_{2}, d_{5}\right)$, where $d_{5}=0.5$. Thus, $d_{4}=1-d_{2}$ and $d_{3}=\sqrt{d_{2}^{2}+d_{5}^{2}}$ The path loss exponent remains at $v=4$, and $P_{p} / \sigma^{2}=20 d B, P_{s} / \sigma^{2}=10 d B, R_{P T}=2 b p s / \mathrm{Hz}, R_{S T}=0.5 \mathrm{bps} / \mathrm{Hz}$.

Fig. 2 shows the optimal power allocation with our proposed spectrum sharing protocol, and the spectrum sharing protocol proposed in [19] where the secondary transmission rate is maximized with only primary QoS guaranteed. We can observe from Fig. 2 that the optimal power allocation $\alpha$ of [19] always equals to $\alpha_{\min }$ which is obtained to guarantee the primary system achieves its target rate. The optimal power allocation $\alpha$ of our proposed spectrum sharing protocol has three different kinds of values. When $d_{2} \in[0.1,051], \alpha$ equals to $\alpha_{\min }$, which is the same as the power allocation in [19]. When $d_{2} \in(0.51,0.89], \alpha$ equals to $\alpha_{\max }$, which is obtained to guarantee the secondary system achieve its target rate. When $d_{2} \in(0.89,1]$, $\alpha$ equals to $\alpha_{0}$, which is obtained to maximized the sum transmission rate of primary and secondary systems.

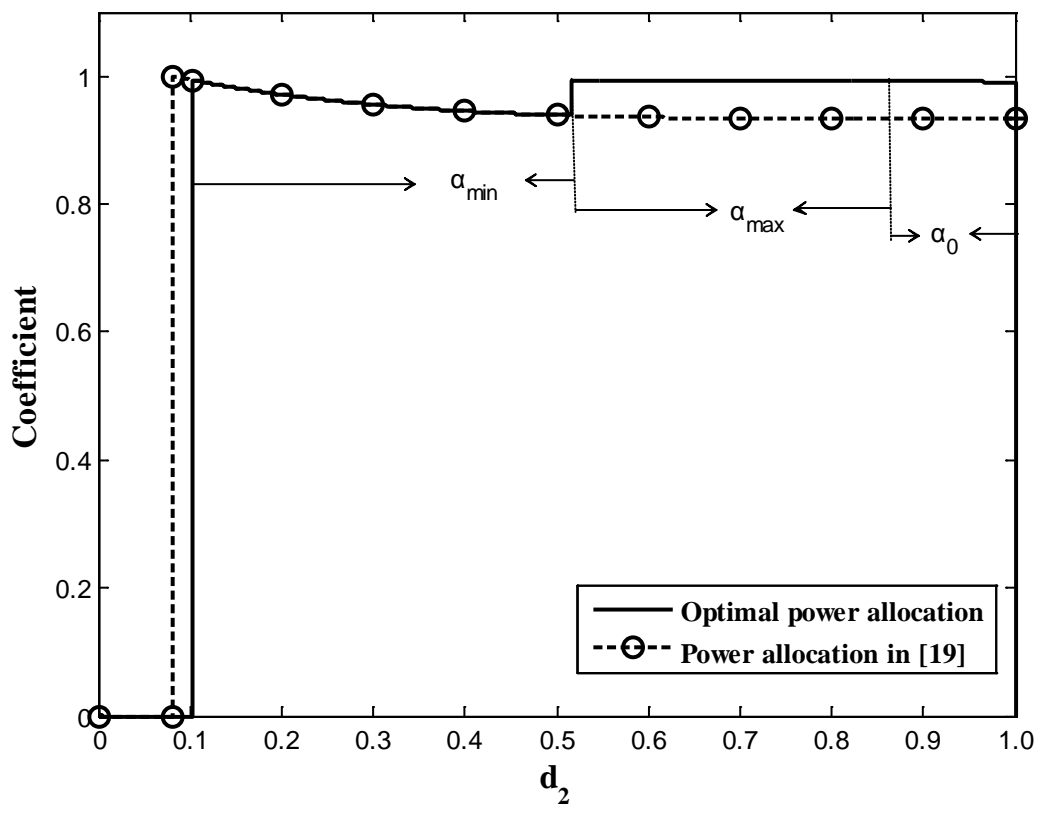

Fig. 2. Optimal power allocation 


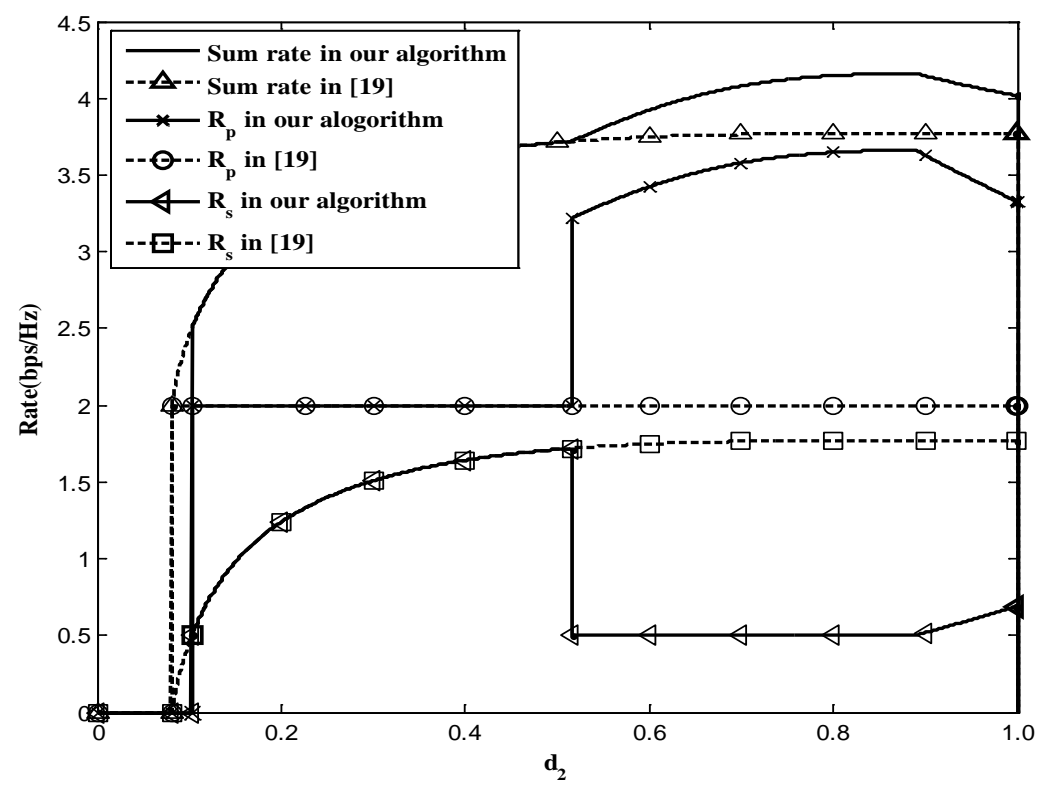

Fig. 3. Achievable rate of primary and secondary systems versus $d_{2}$

Fig. 3 shows the achievable rate of the primary and secondary systems with our proposed spectrum sharing protocol, and the spectrum sharing protocol proposed in [19]. We can observe from Fig. 3 that although the access region of protocol in [19] is a little larger than our protocol. However, we can find that in this larger region, the secondary system cannot achieve its target transmission rate. From Fig. 3 we can also observe that when $d_{2} \in[0.1,1]$, both the primary and secondary system can achieve their target rates in our proposed spectrum sharing protocol and the spectrum sharing protocol proposed in [19]. However, the sum transmission rate of our proposed spectrum sharing protocol is larger than the spectrum sharing protocol proposed in [19].

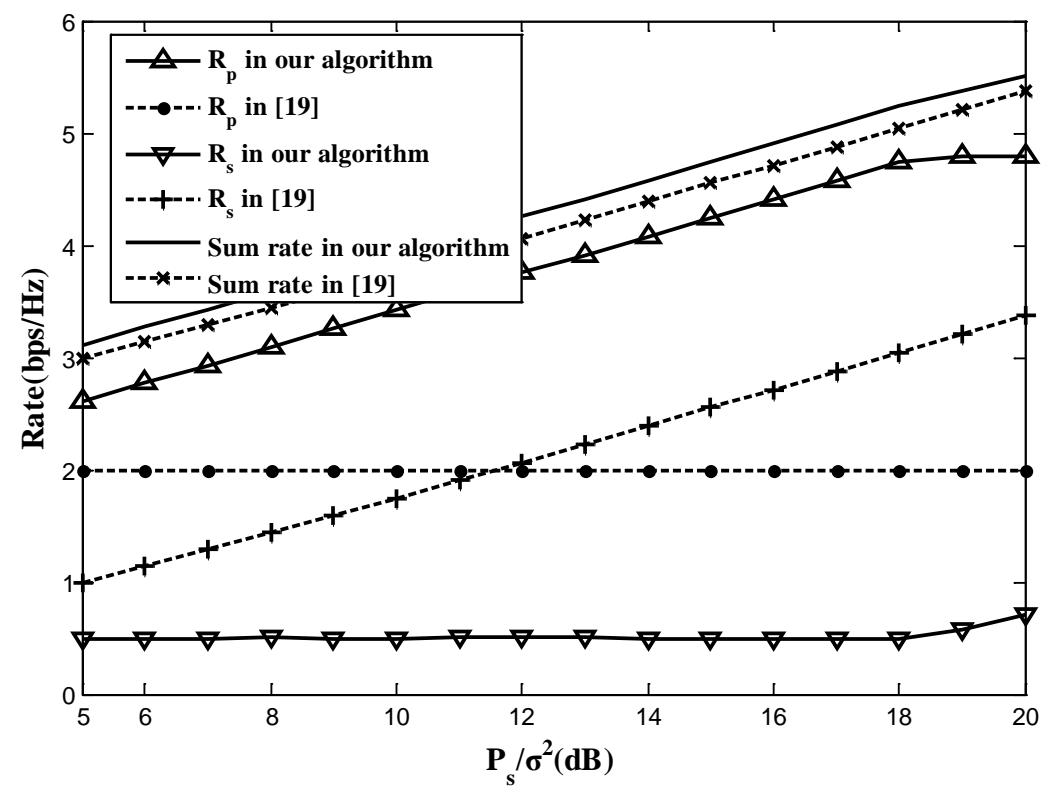

Fig. 4. Achievable rate of primary and secondary systems versus $P_{s} / \sigma^{2}$ 
Fig. 4 shows the achievable rates of the primary and secondary systems with our proposed spectrum sharing protocol, and the spectrum sharing protocol proposed in [19] with different $P_{s} / \sigma^{2}$ when $d_{2}=0.8$. We can find that the sum transmission rate of primary and secondary systems becomes larger with $P_{s} / \sigma^{2}$ increases. We can also observe from Fig. 4 that the sum transmission rate of our proposed protocol is also larger than the protocol proposed in [19] with $P_{s} / \sigma^{2}$ changes.

\section{Conclusion}

In this paper, we propose a sum transmission rate maximization based cooperative spectrum sharing protocol, in which both the primary and secondary systems can achieve their QoS. In the proposed spectrum sharing protocol, the secondary system gains spectrum access by acting as a DF relay to assist the primary system achieve its target rate. Specifically, the selected secondary transmitter helps forward the primary signal by using a fraction power. As a reward, it can use the remaining power to transmit its own signal to achieve the target rate, and thus gain spectrum access. We study the secondary user selection and optimal power allocation such that the sum transmission rate of primary and secondary systems is maximized, while the QoS of both primary and secondary systems can be guaranteed. Simulation results are presented to show that the proposed secondary spectrum access scheme can benefit both primary and secondary systems.

\section{References}

[1] D. A. Roberson, C. S. Hood, J. L. LoCicero, and J. T. MacDonald, "Spectral occupancy and interference studies in support of cognitive radio technology deployment," in Proc. of IEEE Workshop on Networking Technologies for SDR, pp. 26-35, September 2006.

Article (CrossRef Link).

[2] Mark A. McHenry, "Frequency agile spectrum access technologies," in Proc. of FCC Workshop on Cognitive Radios, 2003. Article (CrossRef Link).

[3] J. Mitola and G. Q. Maguire, “Cognitive radios: making software radios more personal,” IEEE Personal Communications, vol. 6, no. 4, pp. 13-18, August 1999. Article (CrossRef Link).

[4] S. Hu, G. A. Bi, Y. L. Guan, and S. Q. Li, "TDCS-based cognitive radio networks with multiuser interference avoidance,” IEEE Transactiom on Communications, vol. 61, no. 12, pp. 4828-4835, December 2013. Article (CrossRef Link).

[5] A.Jovicic and P. Viswanath, "Cognitive radio: an information-theoretic perspective," IEEE Transaction on Information Theory, vol. 55, no. 9, pp. 3945-3958, September 2009. Article (CrossRef Link).

[6] M. C. Asaduzzaman, and M. F. Kader, "Cognitive Radio MAC Protocols: A Survey, Research Issues, and Challenges,” Smart Computing Review, vol. 5, no. 1, pp. 19-29, February 2015. Article (CrossRef Link).

[7] X. Kang, Y.-C. Liang, A. Nallanathan, H. K. Garg, and R. Zhang, “Optimal power allocation for fading channels in cognitive radio networks: Ergodic capacity and outage capacity,” IEEE Transactiom on Communications, vol. 8, pp.940-950, Febrary 2009. Article (CrossRef Link).

[8] A. Ghasemi and E. S. Sousa, "Fundamental limits of spectrum-sharing in fading environments," IEEE Transactiom on Communicatiosn, vol. 6, no. 2, pp. 649-658, Febrary 2007. Article (CrossRef Link). 
[9] N. Zhao and H. J. Sun, "Robust power control for cognitive radio in spectrum underlay networks," KSII Transactions on Internet and Information Systems, vol. 5, no. 7, pp. 1214-1229, July, 2011. Article (CrossRef Link).

[10] M. Hasegawa, H. Hirai, K. Nagano, H. Harada, “Optimization for Centralized and Decentralized Cognitive Radio Networks,” in Proc. of the IEEE, vol. 102, no. 4, pp. 574-584, March 2014. Article (CrossRef Link).

[11] J. W. Li, Z. B. Feng, Z. Y. Feng, P. Zhang, “A survey of security issues in Cognitive Radio Networks,” China Communications, vol. 12, no. 3, pp. 132-152, March 2015. Article (CrossRef Link).

[12] L. Zheng, C. W. Tan, "Maximizing Sum Rates in Cognitive Radio Networks: Convex Relaxation and Global Optimization Algorithms,” IEEE Journal on Selected Areas in Communications, vol. 32, no. 3, pp. 667- 680, March 2014. Article (CrossRef Link).

[13] V. Asghari, and S. Aissa, "Performance of cooperative spectrum-sharing systems with amplify-and-forward relaying,” IEEE Transactiom on Communications, vol. 11, pp. 1295-1300, No. 4, April 2012. Article (CrossRef Link).

[14] J. N. Laneman, D.N. C Tse, and G. W. Wornell, "Cooperative diversity in wireless networks: Efficient protocols and outage behavior,” IEEE Transaction on Information Theory, vol. 50, no. 12, pp.3062-3080, December 2004. Article (CrossRef Link).

[15] M. Naeem, A. Anpalagan, M. Jaseemuddin, D. C. Lee, "Resource Allocation Techniques in Cooperative Cognitive Radio Networks,” IEEE Communication Surveys and Tutorials, vol. 16, no. 2, pp.729-744, May 2014. Article (CrossRef Link).

[16] M.B. Pandian, M. L. Sichitiu, H. Y. Dai, “Optimal Resource Allocation in Random Access Cooperative Cognitive Radio Networks,” IEEE Transaction on Mobil Computing, vol. 14, no. 6, pp.1245-1258, June 2015. Article (CrossRef Link).

[17] W. D. Lu and J. Wang, "Opportunistic Spectrum Sharing Based on Full- Duplex Cooperative OFDM Relaying,” IEEE Communication Letters, vol. 18, no. 2, pp. 241-244, Febrary 2014. Article (CrossRef Link).

[18] Y. Zou, Y. Yao, and B Zheng, "Cooperative relay techniques for cognitive radio systems: Spectrum sensing and secondary user transmissions,” IEEE Communication Magzine., vol. 50, no. 4, pp. 98-103, April 2012. Article (CrossRef Link).

[19] Y. Han, A. Pandharipande, and S. H. Ting, "Cooperative decode and forward relaying for secondary spectrum access,” IEEE Transactiom on Communications, vol. 8, no. 10, pp. 4945-4950, October 2009. Article (CrossRef Link).

[20] S. Singh, P. D. Teal, P. A. Dmochowski, A. J. Coulson, "Robust Cognitive Radio Cooperative Beamforming,” IEEE Transactiom on Communications, vol. 13, no. 11, pp. 6370-6381, November 2014. Article (CrossRef Link).

[21] J. H. Noh, S. J. Oh, "Cognitive Radio Channel with Cooperative Multi-Antenna Secondary Systems," IEEE Journal on Selected Areas in Communications, vol. 32, no. 3, pp. 539-549, March 2014. Article (CrossRef Link). 


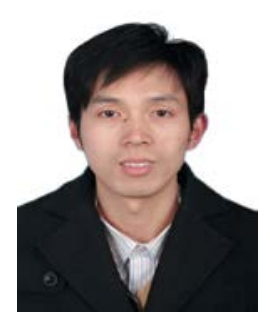

Lu Weidang received the Ph.D. degree in Information and Communication Engineering from Harbin Institute of Technology. He is now with the College of Information Engineering at Zhejiang University of Technology as an assocaite professor. He was a visiting Ph.D. student of Nanyang Technology University. His current research interests include cooperative communications, OFDM, and cognitive radio.
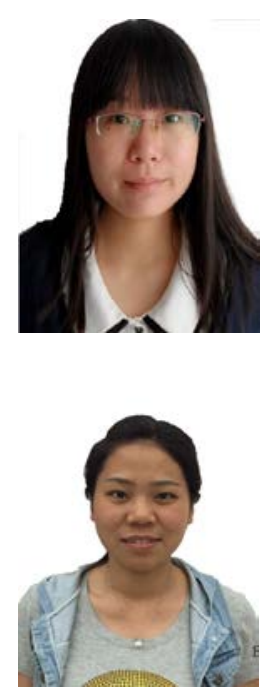

Wang Mengyun is currently a Master candidate at Zhejiang University of Technology, Hangzhou, China. Her current research interests include cognitive radio, OFDM cooperative communications.

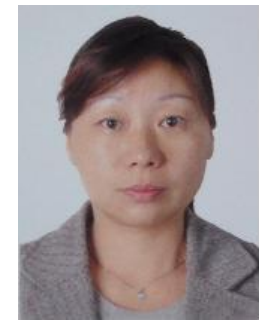

Peng Hong Associate Professor with Zhejiang University of Technology, China. Her research interests include network optimization and cognitive radio.

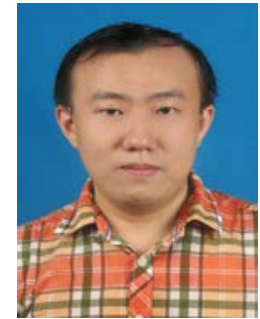

Liu Xin received the Ph.D. degree in Information and Communication Engineering from Harbin Institute of Technology. He is now with Nanjing University of Aeronautics and Astronautics as an assist professor. He was a research fellow of Nanyang Technology University. His current research interests include cooperative communications and cognitive radio Nanjing University of Aeronautics and Astronautics. 